\title{
ARTICLE PK/PD modeling based on NO-ET homeostasis for improving management of sunitinib-induced hypertension in rats
}

\author{
Hao-chen Liu ${ }^{1}$, Xiao-ting Zhou ${ }^{1}$, Yun-si Zheng ${ }^{1}$, Hua He ${ }^{1}$ and Xiao-quan Liu ${ }^{1}$
}

\begin{abstract}
Sunitinib is an oral small molecule multitargeted tyrosine kinase inhibitor, which is currently used to treat severe cancers. Clinical research has shown that patients treated with sunitinib develop hypertension. As soon as sunitinib-induced hypertension appears, it is usual to administer anti-hypertension agent. But this treatment may cause acute blood pressure fluctuation which may lead to additional cardiovascular risk. The aim of this study is to establish a mathematical model for managing sunitinib-induced hypertension and blood pressure fluctuation. A mechanism-based PK/PD model was developed based on animal experiments. Then this model was used to perform simulations, thus to propose an anti-hypertension indication, according to which the antihypertension treatment might yield relative low-level AUC and fluctuation of blood pressure. The simulation results suggest that the anti-hypertension agent may yield low-level AUC and fluctuation of blood pressure when relative ET-1 level ranges from - $15 \%$ to $5 \%$ and relative NO level is more than $10 \%$ compared to control group. Finally, animal experiments were conducted to verify the simulation results. Macitentan $(30 \mathrm{mg} / \mathrm{kg}$ ) was administered based on the above anti-hypertension indication. Compared with the untreated group, the optimized treatment significantly reduced the AUC of blood pressure; meanwhile the fluctuation of blood pressure in optimized treatment group was $70 \%$ less than that in immediate treatment group. This work provides a novel model with potential translational value for managing sunitinib-induced hypertension.
\end{abstract}

Keywords: sunitinib; hypertension; blood pressure fluctuation; PK/PD modeling; simulation; macitentan

Acta Pharmacologica Sinica (2020) 41:719-728; https://doi.org/10.1038/s41401-019-0331-8

\section{INTRODUCTION}

Sunitinib, an oral small-molecule multitargeted tyrosine kinase inhibitor, is currently approved for the treatment of metastatic renal cell carcinoma, imatinib-resistant gastrointestinal stromal tumor (GIST), and pancreatic neuroendocrine tumor [1]. Previous clinical research has shown that patients treated with sunitinib develop hypertension [2]. Hypertension induced by sunitinib is associated with serious cardiovascular complications, such as coronary artery disease (CAD) and heart failure, and the use of vascular endothelial growth factor (VEGF) inhibition may be compromised in cancer patients who develop hypertension $[3,4]$. Improving the management of hypertension caused by sunitinib may be helpful in preventing severe cardiotoxicity. However, randomized controlled trials on the treatment of angiogenesis inhibition-induced hypertension are not available, and no clear recommendation for a particular antihypertensive agent or class agents can be given [5]. The clinical use of sunitinib may be compromised without a suitable antihypertension strategy. To address this challenge, preclinical studies on suitable antihypertensive strategies should be performed.

Previous preclinical studies have investigated the use of antihypertensive therapy for hypertension induced by sunitinib $[3,6-8]$. These studies identified treatments for inhibiting hypertension induced by sunitinib, but the fluctuation of blood pressure during antihypertensive treatment was ignored in these studies. In the previous studies, sunitinib and antihypertensive agents were administered simultaneously, which may have yielded dramatic fluctuations in blood pressure; e.g., the antihypertensive treatment proposed by Witte et al. doubled blood pressure fluctuation levels compared to that in subjects that did not receive antihypertensive treatment. The treatment protocol reported by Lankhorst et al. resulted in a similar fluctuation, but such a fluctuation was not observed in subjects that did not receive antihypertensive treatment [3, 8]. Previous studies have revealed that blood pressure variability increases cardiac, vascular, and renal damage $[9,10]$. Therefore, the high blood pressure variability induced by antihypertensive treatment may limit the benefits provided by lowering blood pressure. The aim of this study was to develop a mechanismbased pharmacokinetics-pharmacodynamic (PK/PD) model for proposing an optimized antihypertensive management method that can lower both blood pressure and its fluctuation in rats.

In this study, a mechanism-based PK-PD model was developed to optimize antihypertensive treatment. It is crucial to understand the pathophysiological mechanisms of hypertension induced by sunitinib to develop such a model. Sunitinib acts by inhibiting vascular endothelial growth factor receptors (VEGFR-1, VEGFR-2, and VEGFR-3), platelet-derived growth factor receptors (PDGFR- $\alpha$ and PDGFR- $\beta$ ), stem cell factor receptor (KIT), Fms-like tyrosine kinase-3 receptor (FLT3), colony stimulating factor receptor type 1 (CSF-1 R), and the glial cell linederived neurotrophic factor receptor (RET) [11]. Among these

${ }^{1}$ Center of Drug Metabolism and Pharmacokinetics, China Pharmaceutical University, Nanjing 210009, China

Correspondence: Hua He (huahe_cpupk@cpu.edu.cn) or Xiao-quan Liu (Ixq@cpu.edu.cn)

Received: 22 July 2019 Accepted: 4 November 2019

Published online: 13 January 2020 
targets of sunitinib, the VEGF pathway is frequently associated with the development of severe hypertension [5]. Sunitinib can decrease NO production by inhibiting the VEGF pathway, which plays a key role in blood pressure regulation [5]. Moreover, previous studies have reported that sunitinib can elevate the plasma level of endothelin-1 (ET-1), the most potent vasoconstrictor identified thus far $[5,7,12]$. In summary, the mechanism of hypertension induced by sunitinib mainly involves two pathways. First, sunitinib inhibits vasodilation by decreasing NO production. Second, sunitinib induces vasoconstriction by elevating ET-1 levels.

In this study, based on the mechanisms mentioned above, a mechanism-based PK-PD model was developed. The present study was performed in four parts. First, animal experiments were performed to collect data for PK-PD modeling. Second, a mechanism-based PK-PD that can provide insight into the relationship between the plasma concentration of sunitinib and blood pressure was developed. Third, simulations based on the PK-PD model and progression analysis were performed according to the proposed hypothesis that the effect of antihypertensive treatment on blood pressure and its fluctuation are affected by variations in NO-ET homeostasis. The simulations demonstrated the different effects of antihypertensive treatment on blood pressure and its fluctuation under different states of NO-ET homeostasis. Fourth, animal experiments were performed to verify the antihypertensive treatment proposed by the PK-PD model. Our study provides a mechanism-based model to optimize an antihypertensive treatment for hypertension induced by sunitinib that not only inhibits hypertension but also reduces blood pressure fluctuations in rats.

\section{MATERIALS AND METHODS}

Animal experiments

Male Wistar Kyoto rats (220-250 g), obtained from Super-B\&K Laboratory Animal Center (Shanghai, China), were housed at $22 \pm$ $5^{\circ} \mathrm{C}$ on a 12 -h dark/light cycle and given access to standard laboratory rat chow and water ad libitum. All animal experiments were approved by the Animal Ethics Committee of China Pharmaceutical University. All experiments were performed in accordance with relevant guidelines and regulations.

In this study, an animal experiment was performed to obtain data for the development of a PK-PD model. In this experiment, rats were randomly administered sunitinib $(26.7 \mathrm{mg} / \mathrm{kg}$ per day; $n=6)$ or vehicle $(n=6)$ by oral gavage for 10 days. The dosage of sunitinib was based on previous experimental studies that investigated hypertension induced by sunitinib $[3,7,13,14]$. The protocol for systolic blood pressure (SBP) measurement was designed based on the method described by Whitesall et al. and Kubota et al. $[15,16]$. All rats were acclimated to restraint, tailcuff inflation and heating the day before the start of the experiment. The rats were placed in plastic restrainers with heating pads set to $33 \sim 34{ }^{\circ} \mathrm{C}$. The instrument (ALC-NIBP, ALCBIO; Shanghai, China) automatically took ten 30-s measurements. SBP values were recorded when more than five consecutive stable readings were available. The highest and lowest readings were discarded, and the remaining readings were averaged to obtain one data point. SBP was measured daily. Blood samples were collected in tubes containing ethylene diamine tetraacetic acid (EDTA) daily. Samples were collected at the same time on each sampling day. Plasma was prepared by centrifugation at $4000 \times g$ and $4^{\circ} \mathrm{C}$ for $10 \mathrm{~min}$. The prepared plasma samples were stored at $-70^{\circ} \mathrm{C}$ until analysis.

\section{Drug analysis}

The plasma concentration of sunitinib was determined by LC-MS/MS according to the method described in previous studies with slight modifications $[17,18]$. A total of $30 \mu \mathrm{L}$ of plasma was mixed with $5 \mu \mathrm{L}$ IS working solution $\left(100 \mathrm{ng} \cdot \mathrm{mL}^{-1}\right.$ paliperidone in water). After vortex-mixing, $1 \mathrm{~mL}$ of acetonitrile was added to the mixture to precipitate the proteins. Then, the mixture was centrifuged for $10 \mathrm{~min}$ at $15000 \mathrm{r} / \mathrm{min}$ at $4{ }^{\circ} \mathrm{C}$. A total of $50 \mu \mathrm{L}$ of the supernatant was diluted with $100 \mu \mathrm{L}$ of water for analysis.

The prepared samples were injected into a Shimadzu ODS column $(5.0 \mu \mathrm{m}, 150 \mathrm{~mm} \times 2.0 \mathrm{~mm})$. The mobile phase consisted of acetonitrile containing $0.1 \%$ formic acid (phase A) and water containing $0.1 \%$ formic acid (phase $\mathrm{B}$ ). The flow rate of the mobile phase was $0.2 \mathrm{~mL} \cdot \mathrm{min}^{-1}$, and gradient elution was used (0-1.0 $\mathrm{min}, 80 \%$ phase $\mathrm{B} ; 1.0-2.2 \mathrm{~min}, 80 \%-45 \%$ phase $\mathrm{B} ; 2.2-5.3$ min, $45 \%$ phase $B ; 5.3-6.5 \mathrm{~min}, 45 \%-80 \%$ phase $B$; $6.5-9.0 \mathrm{~min}$, $80 \% \mathrm{~B}$ ). The injection volume was $5 \mu \mathrm{L}$. The column oven was set to $+40^{\circ} \mathrm{C}$. A TSQ Quantum triple quadrupole mass spectrometer (Thermo Fisher, American) using selected reaction monitoring (SRM) mode and an electrospray ionization source (ESI) in positive ion mode was utilized to obtain mass spectra at a voltage of $4000 \mathrm{~V}$. The sheath gas pressure and auxiliary gas pressure were maintained at $30 \mathrm{~L} \cdot \mathrm{min}^{-1}$ and $20 \mathrm{~L} \cdot \mathrm{min}^{-1}$, respectively. The capillary temperature was maintained at $380^{\circ} \mathrm{C}$ throughout the run. The collision energy for the analyte and IS was $26 \mathrm{eV}$. The precursor to product ion transition (Q1 to Q3) for the quantitation $(\mathrm{m} / \mathrm{z})$ of sunitinib and IS were programmed in the spectrometer as 399.1 to 283.0 and 427.2 to 207.1 , respectively.

Biomarker analysis

Plasma endothelin-1 (ET-1) was measured using radioimmunoassay with a commercial kit obtained from Beijing North Institute of Biological Technology (Beijing, China).

Plasma NO levels are difficult to determine. Therefore, the levels of the stable end products of NO radicals, nitrite and nitrate, were determined to measure the production of $\mathrm{NO}$ radicals based on the method described in a report of Han Moshage [19]. Fifty microliters of plasma was diluted with $25 \mu \mathrm{L}$ of distilled water. Then, $15 \mu \mathrm{L}$ of nitrate reductase, $10 \mu \mathrm{L}$ of $\mathrm{NADPH}$, and $2 \mu \mathrm{L}$ of FAD were added to the samples to yield final concentrations of $200 \mathrm{U} / \mathrm{L}, 50 \mu \mathrm{mol} / \mathrm{L}$ and $5 \mu \mathrm{mol} / \mathrm{L}$ respectively. The samples were subsequently incubated for $20 \mathrm{~min}$ at $37^{\circ} \mathrm{C}$ and then mixed with $10 \mu \mathrm{L}$ of lactate dehydrogenase at a final concentration of $10 \mathrm{mg} / \mathrm{L}$ and $10 \mu \mathrm{L}$ of sodium pyruvate at a final concentration of $10 \mathrm{mmol} / \mathrm{L}$. The samples were further incubated for $5 \mathrm{~min}$ at $37^{\circ} \mathrm{C}$ to oxidize NADPH. Ten microliters of zinc sulfate solution $(0.38 \mathrm{~g} / \mathrm{mL})$ was mixed with the samples. After vortex-mixing, the mixture was centrifuged for $5 \mathrm{~min}$ at $10000 \mathrm{r} / \mathrm{min}$. Then, $100 \mu \mathrm{L}$ of the supernatant and $100 \mu \mathrm{L}$ of Griess reagent ( $1 \mathrm{~g} / \mathrm{L}$ sulfanilamide, $25 \mathrm{~g} / \mathrm{L}$ phosphoric acid, and $0.1 \mathrm{~g} / \mathrm{L} \mathrm{N}$-1-naphthylethylenediamine) were added to a microtiter plate well. After $10 \mathrm{~min}$ of color development at room temperature, the absorbance was measured on a microplate reader at a wavelength of $540 \mathrm{~nm}$.

Creatinine was measured with a commercial kit obtained from Nanjing Jiancheng Bioengineering Institute (Nanjing, China).

\section{Model development}

The process of model development is shown in Fig. 1. First, a mechanism-based PK-PD model was developed based on animal experiments. Second, based on the PK-PD model, simulations were performed to investigate the potential appropriate antihypertensive methods. Finally, the results of the simulations were verified by animal experiments.

\section{PK-PD model}

The PK parameters of sunitinib were estimated based on a single-dose PK profile reported by Speed et al. [20]. In this study, rats were administered sunitinib $(6 \mathrm{mg} / \mathrm{kg}$ or $15 \mathrm{mg} / \mathrm{kg}$ ) by oral gavage, and then the plasma concentration was determined 1, $3,6,8,24,48$, and $72 \mathrm{~h}$ after oral administration. Then, the 


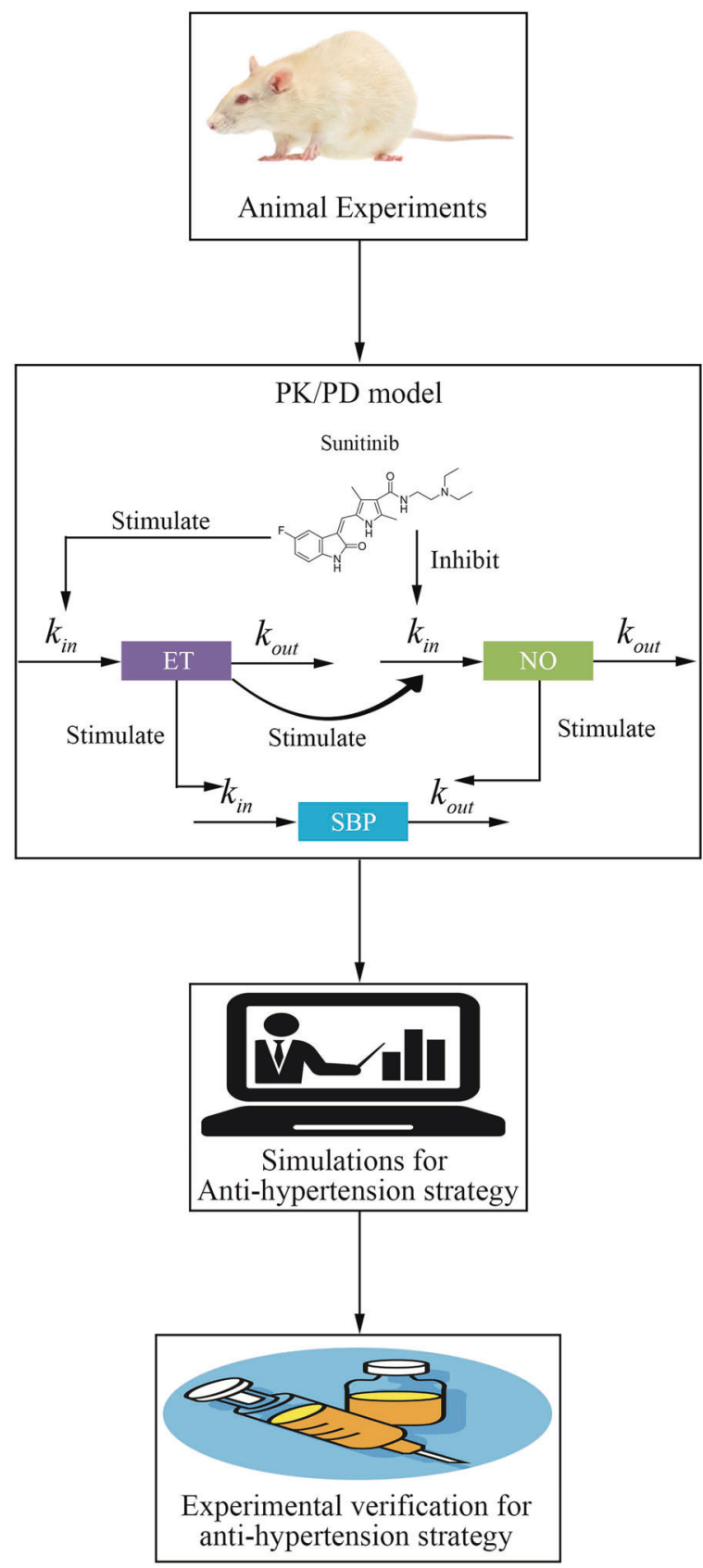

Fig. 1 The structure of the model-based research framework.

sunitinib plasma concentration was simulated based on the above parameters after multi-dose administration. Eq. 1 was used to estimate the time course of sunitinib concentration in the plasma. $k_{a}^{\text {sunitinib }}$ represents the absorption rate constant of sunitinib. $V_{\text {sunitinib }}$ represents the apparent volume of distribution. $x_{0}$ represents the dose of sunitinib. $k_{\text {out }}^{\text {sunitib }}$ represents the elimination rate constant.

$$
\begin{aligned}
& c_{\text {sunitinib }}= \\
& \frac{k_{a}^{\text {sunitinib }} x_{0}}{V_{\text {sunitinib }}\left(k_{a}^{\text {sunitinib }}-k_{\text {out }}^{\text {sunitinib }}\right)}\left(e^{-k_{\text {out }}^{\text {sunitinib }} t}-e^{-k_{a}^{\text {sunitinib }} t}\right)
\end{aligned}
$$

The mechanism-based pharmacodynamics model was described in the system, which was composed of three linked turnover equations:

$$
\begin{aligned}
& \frac{\mathrm{d} c_{\mathrm{NO}}}{\mathrm{dt}}=k_{\mathrm{in}}^{\mathrm{NO}}\left(E_{\mathrm{ET}}-k_{1} c_{\text {sunitinib }}\right)-k_{\text {out }}^{\mathrm{NO}} c_{\mathrm{NO}} \\
& \frac{\mathrm{d} c_{\mathrm{ET}}}{\mathrm{dt}}=k_{\mathrm{in}}^{\mathrm{ET}}\left(1+k_{2} c_{\text {sunitinib }}\right)-k_{\text {out }}^{\mathrm{ET}} c_{\mathrm{ET}} \\
& \frac{\mathrm{dSBP}}{\mathrm{dt}}=k_{\mathrm{in}}^{\mathrm{SBP}}\left(1+k_{3} c_{\mathrm{ET}}\right)-k_{\text {out }}^{\mathrm{SBP}}\left(1+\mathrm{SBP}+\mathrm{k}_{4} \mathrm{c}_{\mathrm{NO}}\right)
\end{aligned}
$$

The basal turnover of NO was determined by a zero-order production rate $\left(k_{\mathrm{in}}^{\mathrm{NO}}\right)$ and a first-order degradation rate $\left(k_{\mathrm{out}}^{\mathrm{NO}}\right)$. In this system, the production of NO may be affected by ET-1 and sunitinib. On one hand, sunitinib may decrease NO production by reducing the expression of endothelial nitric oxide synthase (eNOS) and neuronal nitric oxide synthase (nNOS) [5, 21, 22]. In this study, the inhibition of NO production induced by sunitinib was assumed to be linear and concentration-dependent and was described by parameter $k_{1}$. On the other hand, ET-1 may indirectly decrease the thick ascending limb of the loop of Henle (TALH) by activating ETB receptors, increasing intracellular calcium concentration, and stimulating NO release [23]. In this study, the effect of ET-1 on stimulating NO release was assumed to be constant $\left(E_{\mathrm{ET}}\right)$. The basal turnover of ET-1 was determined by a zero-order production rate $\left(k_{\text {in }}^{\mathrm{ET}}\right)$ and a first-order degradation rate $\left(k_{\text {out }}^{\mathrm{ET}}\right)$. Sunitinib may elevate ET-1 [5]. In this study, the sunitinib-induced increase in ET-1 was assumed to be linear and concentration-dependent and was described by parameter $k_{2}$. The basal turnover of SBP was determined by a zero-order production rate $\left(k_{\text {in }}^{\mathrm{SBP}}\right)$ and a first-order degradation rate $\left(k_{\text {out }}^{\mathrm{SBP}}\right)$. Both ET-1 and NO play important roles in blood pressure. ET-1 may induce vasoconstriction and elevate blood pressure. The effect of ET-1 on blood pressure was assumed to be linear and concentration-dependent and was described by parameter $k_{3}$. NO may induce vasodilatation and reduce blood pressure. The effect of NO on blood pressure was assumed to be linear and concentration-dependent and was described by parameter $k_{4}$. All the assumptions of the PK/PD model were proposed based on the experimental data used to acquire a parsimonious model with relatively good fitness.

\section{Simulations}

Previous research has demonstrated that amlodipine and macitentan can help reduce blood pressure [3]. In this study, amlodipine and macitentan were considered candidate agents. The simulations were performed based on the PK-PD model. The equation system used for the simulations contained three equations. The first two equations in this system were the same as Eqs. 2, 3. As the anti-hypertension mechanisms of amlodipine and macitentan are different, the third equations for amlodipine and macitentan were different and were as follows:

$$
\frac{\mathrm{dSBP}}{\mathrm{dt}}=k_{\mathrm{in}}^{\mathrm{SBP}}\left(1+k_{3} \mathrm{C}_{\mathrm{ET}}\right)-k_{\text {out }}^{\mathrm{SBP}}\left(1+\mathrm{SBP}+\mathrm{k}_{4} \mathrm{C}_{\mathrm{NO}}+\mathrm{E}_{\text {amlodipine }}\right)
$$

$E_{\text {amlodipine }}=c_{\text {amlodipine }} \frac{\left(\operatorname{Tr}_{\text {amlodipine }} \mathrm{t}\right)^{n_{\text {amlodipine }}}}{n_{\text {amlodipine }} !} e^{-\operatorname{Tr}_{\text {amlodipine }} \mathrm{t}}$

$\frac{\mathrm{dSBP}}{\mathrm{dt}}=k_{\mathrm{in}}^{\mathrm{SBP}}\left(1+k_{3} \mathrm{C}_{\mathrm{ET}}-E_{\text {macitentan }}\right)-k_{\text {out }}^{\mathrm{SBP}}\left(1+\mathrm{SBP}+\mathrm{k}_{4} \mathrm{C}_{\mathrm{NO}}\right)$

$E_{\text {macitentan }}=c_{\text {macitentan }} \frac{\left(\operatorname{Tr}_{\text {macitentan }}\right)^{n_{\text {macitentan }}}}{n_{\text {macitentan }} !} e^{-\operatorname{Tr}_{\text {macitentan }} t}$ 
Equations 5, 6 were used to simulate the effect of amlodipine. $E_{\text {amlodipine }}$ represents the effect of amlodipine. $C_{\text {amlodipine }}$ is the plasma concentration of amlodipine. $C_{\text {amlodipine }}$ was obtained by simulating the multidose administration of amlodipine based on a first-order absorption one-compartment model whose parameters are estimated according to the data extracted from a previous experimental report [24]. $n_{\text {amlodipine }}$ is the number of transit compartments of amlodipine. $\operatorname{Tr}_{\text {amlodipine }}$ stands for a transit rate constant between transit compartments of amlodipine. $n_{\text {amlodipine }}$ and $\mathrm{Tr}_{\text {amlodipine }}$ were estimated based on the data extracted from a previous experimental report [3]. Equations 7, 8 were used to simulate the effect of macitentan. $E_{\text {macitentan }}$ represents the effect of amlodipine. $c_{\text {macitentan }}$ is the plasma concentration of macitentan. $c_{\text {macitentan }}$ was obtained by simulating the multidose administration of macitentan based on a first-order absorption one-compartment model whose parameters are estimated according to the data extracted from a previous experimental report [25]. $n_{\text {macitentan }}$ is the number of transit compartments of amlodipine. Trmacitentan stands for a transit rate constant between transit compartments of amlodipine. $n_{\text {macitentan }}$ and $\operatorname{Tr}_{\text {macitentan }}$ were estimated based on the data extracted from a previous experimental report [3]. Parameters $n_{\text {amlodipine, }} \operatorname{Tr}_{\text {amlodipine, }} n_{\text {macitentan, }}, \operatorname{Tr}_{\text {macitentan }}$ and the pharmacokinetic parameters of amlodipine and macitentan are listed in Table 1.

In the simulation, the antihypertensive effect was represented by the area under the curve (AUC) of blood pressure, and the average real variability (ARV) was used to measure the fluctuation of blood pressure (Eq. 9) [26]. In Eq. 9, $N$ denotes the number of valid blood pressure (BP) measurements. To avoid bias based on background variation in the simulation, the percentage change in the ARV compared to that of the control group ( $\triangle A R V$, Eq. 10) was used in the simulation. The simulation was performed based on three scenarios. The aim of scenario I was to choose a suitable antihypertensive agent. Therefore, amlodipine and macitentan were administered at different time points (day 0 to day 9,9 time points in total) after sunitinib was administered. Then, the AUC of blood pressure and $\triangle A R V$ yielded by amlodipine and macitentan treatment were compared to find a suitable antihypertensive agent. The dose of amlodipine was set to $1 \mathrm{mg} / \mathrm{kg}$ (equivalent to the maximum tolerated dose in humans), and the dose of macitentan was set to $30 \mathrm{mg} / \mathrm{kg}$ (equivalent to a high but well tolerated dose in humans) $[27,28]$. The aim of scenario II was to find the appropriate dose of the selected antihypertensive agent. For this purpose, different doses of selected antihypertensive agents were administered. Then, the AUC of blood pressure and $\triangle A R V$ were calculated. The aim of scenario III was to investigate the indication for antihypertensive treatment. For this purpose, the proposed antihypertensive agent was administered under different levels of ET and NO. Then, the AUC of blood pressure and $\triangle A R V$ were calculated. The relative levels of both $\mathrm{NO}$ and $\mathrm{ET}$ in the control group changed from -50 to $50 \%$. A total of 2500 situations were simulated. The dose of sunitinib was $26.5 \mathrm{mg} / \mathrm{kg}$, and the dose of

Table 1. PK-PD parameters of amlodipine and macitentan for simulation.

\begin{tabular}{lcllll}
\hline & $k_{a}\left(\mathrm{~h}^{-1}\right)$ & $V(\mathrm{~L})$ & $k_{\text {out }}\left(\mathrm{h}^{-1}\right)$ & $n$ & $\operatorname{Tr}(\mathrm{h})$ \\
\hline Amlodipine & 13.92 & 4.39 & 0.022 & 1 & 0.32 \\
Macitentan & 0.22 & 42628.85 & 0.044 & 1 & 0.45 \\
\hline
\end{tabular}

$k_{a}$ first-order absorption rate constant, $V$ apparent volume of distribution, $k_{\text {out }}$ elimination rate constant, $n$ number of transit compartments, $\operatorname{Tr}$ transit rate constant between transit compartments macitentan was selected according to scenario II.

$$
\begin{aligned}
& \mathrm{ARV}=\frac{1}{N-1} \sum_{k=1}^{N-1}\left|\mathrm{BP}_{\mathrm{k}+1}-\mathrm{BP}_{\mathrm{k}}\right| \\
& \Delta \mathrm{ARV}=\frac{\mathrm{ARV}_{\text {Treated }}-\mathrm{ARV}_{\text {Control }}}{\mathrm{ARV}_{\text {Control }}}
\end{aligned}
$$

\section{Validation of modeling and simulation}

The PK model was validated by two pieces of data. First, it was validated by the plasma concentration after a single dose of sunitinib $(6 \mathrm{mg} / \mathrm{kg}$ ) was administered [20]. Second, it was validated by the trough concentration after multiple doses of sunitinib (26.5 $\mathrm{mg} / \mathrm{kg}$ and $10 \mathrm{mg} / \mathrm{kg}$ ) were administered.

An animal experiment was performed as an experimental validation for the PK/PD model. In this experiment, rats were randomly administered low-dose sunitinib $(10 \mathrm{mg} / \mathrm{kg}$; once daily; $n=6)$ or vehicle $(n=6)$ by oral gavage for 10 days. The protocol of blood pressure measurement was the same as that used in the first experiment. Blood samples were collected in tubes containing EDTA on days $0,2,4,6,8$, and 9 . The protocols for blood sample preparation and storage were the same as those used in the first experiment.

The proposed antihypertensive method was also verified by another animal experiment. In this animal experiment, 30 male Wistar Kyoto rats $(220-250 \mathrm{~g})$ were randomly divided into five groups. The first group was the control group (given vehicle; $n=6$ ). The second group (the sunitinib group) was orally administered sunitinib ( $26.7 \mathrm{mg} / \mathrm{kg} ; n=6)$ once daily for 10 days. The third group (the low-dose immediate treatment group; $n=6$ ) was orally and simultaneously administered sunitinib $(26.7 \mathrm{mg} / \mathrm{kg})$ and macitentan $(10 \mathrm{mg} / \mathrm{kg})$. The fourth group (the high-dose immediate treatment group; $n=6$ ) was orally and simultaneously administered sunitinib $(26.7 \mathrm{mg} / \mathrm{kg})$ and macitentan $(30 \mathrm{mg} / \mathrm{kg})$. The fifth group (the low-dose optimized treatment group; $n=6$ ) was orally administered sunitinib $(26.7 \mathrm{mg} / \mathrm{kg})$, and then macitentan $(10 \mathrm{mg} / \mathrm{kg})$ was given until the NO and ET levels reached their critical values. The sixth group (the high-dose optimized treatment group; $n=6$ ) was orally administered sunitinib (26.7 $\mathrm{mg} / \mathrm{kg})$, and then macitentan $(30 \mathrm{mg} / \mathrm{kg})$ was given until the NO and ET levels reached their critical values. The antihypertensive agent, the dosage of macitentan and the critical values of ET and NO levels were selected according to the simulation based on the PK-PD model. The detailed selection procedure are described in the "Materials and methods" and "Results" sections. The protocols for the measurements of SBP, NO and ET were the same as those used in the first experiment. Macitentan was dissolved in vehicle containing $0.5 \%$ methylcellulose aqueous solution and $1 \%$ Tween 80 .

\section{RESULTS}

Biomarker variation

The results of the animal experiments are shown in Fig. 2. The SBP level increased rapidly after sunitinib administration in the highdose group. The SBP level in the low-dose group was elevated 1 day after sunitinib administration. The variation in the SBP level was dose-dependent. The level of NO increased from day 0 to day 2 and decreased from day 3 to day 10 in the high-dose group. In the low-dose group, NO was elevated at 2 days. The ET level increased continuously and dose-dependently in both the highdose group and the low-dose group.

\section{PK-PD model fitting}

The PK-PD parameters were estimated based on the experiment that used high-dose sunitinib (the first experiment). In this 

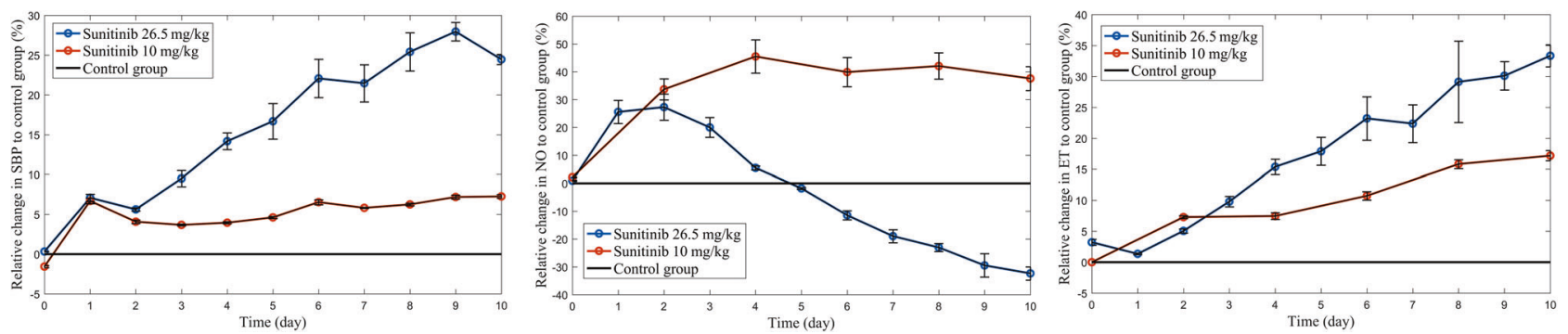

Fig. 2 The variation in SBP, NO, and ET. The variation in biomarkers is presented as the level relative to that of the control group. The black line in the figures represents the control group, which was normalized to 0 . The red line represents the low-dose group. The blue line represents the high-dose group.

\begin{tabular}{|llllll|}
\hline Table 2. & PK-PD model parameters and bootstrap validation. \\
\hline Parameters & Estimate & $\mathrm{CV}(\%)$ & $\mathrm{Cl}$ & \multicolumn{1}{l}{ Bootstrap } \\
\cline { 3 - 6 } & & & $\mathrm{UL}$ & $\mathrm{LL}$ & \\
\cline { 4 - 6 }$k_{a}^{\text {sunitinib }}$ & 1.14 & $44.74 \%$ & 0.63 & 1.65 & - \\
$V_{\text {sunitinib }}$ & 87821.75 & $0.78 \%$ & 87134.05 & 88509.45 & - \\
$k_{\text {out }}^{\text {sunitib }}$ & 0.019 & $5.26 \%$ & 0.018 & 0.02 & - \\
$k_{\text {in }}^{\text {NO }}$ & 0.0047 & $10.64 \%$ & 0.0042 & 0.0051 & 0.0045 \\
$E_{\mathrm{ET}}$ & 93.38 & $1.25 \%$ & 92.21 & 94.55 & 92.76 \\
$k_{1}$ & 250.04 & $0.18 \%$ & 249.6 & 250.48 & 251.41 \\
$k_{\text {out }}^{\text {NO }}$ & 0.44 & $15.91 \%$ & 0.37 & 0.5 & 0.41 \\
$k_{\text {in }}^{\mathrm{ET}}$ & $9.71 \times 10^{-5}$ & $35.75 \%$ & $6.24 \times 10^{-5}$ & $1.31 \times 10^{-4}$ & $1.79 \times 10^{-4}$ \\
$k_{2}$ & 1349.4 & $5.34 \%$ & 1277.39 & 1421.46 & 1349.4 \\
$k_{\text {out }}^{\mathrm{ET}}$ & 0.13 & $84.62 \%$ & 0.02 & 0.25 & 0.28 \\
$k_{\text {in }}^{\text {SBP }}$ & 10.84 & $4.89 \%$ & 10.31 & 11.36 & 10.72 \\
$k_{3}$ & 0.23 & $4.35 \%$ & 0.22 & 0.24 & 0.23 \\
$k_{4}$ & 0.23 & $4.35 \%$ & 0.22 & 0.24 & 0.23 \\
$k_{\text {out }}^{\text {SBP }}$ & 9.69 & $4.64 \%$ & 9.24 & 10.13 & 9.59 \\
\hline
\end{tabular}

experiment, rats were randomly administered sunitinib $(26.7 \mathrm{mg} / \mathrm{kg}$; once daily; $n=6)$ or vehicle $(n=6)$ by oral gavage for 10 days. The estimated PK-PD model parameters are listed in Table 2. Random effects for parameters $E_{\mathrm{ET}}, k_{1}, k_{2}$, and $k_{\mathrm{out}}^{\mathrm{SBP}}$ were induced. The bootstrapping values of these parameters remained near the final parameter estimation with a relatively low coefficient of variance (CV). Goodness-of-fit plots of the final model are shown in Fig. $3 a-c$ and suggest that the errors of both population and individual profiles were acceptable. The visual predictive check (VPC) for the PK-PD model is shown in Fig. 3c. The VPC plots show that the observed average data fell within the $95 \%$ prediction confidence interval. Moreover, a low-dose experiment was performed as an external verification of the PK-PD model. In this experiment, rats were randomly administered sunitinib $(10 \mathrm{mg} / \mathrm{kg}$; once daily; $n=6)$ or vehicle $(n=6)$ by oral gavage for 10 days. The experiment was used to simulate changes in NO, ET, and SBP to verify the PK-PD model, and the simulation results were compared with the experimental data. The results are shown in Fig. 4a, b. The results suggest that the error was acceptable and that the PK-PD model fit the low-dose experiment data. Furthermore, as sunitinib may induce renal damage, which may affect SBP and bias the PKPD model, creatinine levels in the low-dose group, high-dose group and control group were measured to determine whether the model was biased by renal damage. The results are shown in Fig. 4c. The results show that creatinine levels were not significantly different among these three groups at any of the time points ( $P>0.05$, based on one-way ANOVA), suggesting that no significant renal damage occurred during the experiment. Therefore, the goodness of fit of the PK-PD model was satisfactory, and the model was not biased by the renal damage induced by sunitinib.

\section{Simulation}

The results of scenario I simulation are shown in Fig. 5a, b. The simulation suggested that macitentan yielded similar blood pressure fluctuations but lower AUC of blood pressure compared to those induced by amlodipine. Therefore, macitentan was chosen as the antihypertensive agent. The result of scenario II simulation is shown in Fig. $5 \mathrm{c}$. The simulation suggested that the blood pressure fluctuation decreased until the dose of macitentan increased to $40 \mathrm{mg} / \mathrm{kg}$ and that if the dose of macitentan exceeded $40 \mathrm{mg} / \mathrm{kg}$, blood pressure fluctuation increased. The AUC of blood pressure decreased as the dose of macitentan increased. Thus, $40 \mathrm{mg} / \mathrm{kg}$ macitentan should be selected for hypertension treatment. However, $40 \mathrm{mg} / \mathrm{kg}$ macitentan may not be well tolerated (the maximum tolerated dose of macitentan is equivalent to $30 \mathrm{mg} / \mathrm{kg}$ in rats.) [28]. Therefore, $30 \mathrm{mg} / \mathrm{kg}$ macitentan rather than $40 \mathrm{mg} / \mathrm{kg}$ macitentan was selected for hypertension treatment.

The results of scenario III simulation are shown in Fig. 6a-c. We defined low-level AUC as an AUC of blood pressure in the treated group lower than $70 \%$ of that in untreated the group ( $A U C_{\text {Treated }}$ / AUC Untreated $\leq 70 \%$ ) and low-level $\triangle A R V$ as a fluctuation of blood pressure in the treated group lower than that in the untreated group ( $\left.\triangle A R V_{\text {Treated }}<\Delta A R V_{\text {Untreated }}\right)$. According to the response to the antihypertensive agent, there were four states of NO-ET homeostasis. The first state, termed the compensatory unbalanced state (Fig. 6c), was defined as an ET level relative to that of the control group of less than $-15 \%$. The second state, termed the weak balance state, was defined as a relative ET level ranging from $-15 \%$ to $5 \%$ and a relative NO level greater than $10 \%$. The third state, termed the pathological balance state, was defined as a relative ET level ranging from $5 \%$ to $35 \%$ and a relative NO level greater than $10 \%$. The fourth state, termed the pathological unbalanced state, was defined as a relative ET level greater than $35 \%$. If NO-ET homeostasis is in the compensatory unbalanced state, antihypertensive treatment may yield low-level AUC and high-level $\triangle A R V$. If NO-ET homeostasis is in the weak balance zone, antihypertensive treatment may yield low-level AUC and low-level $\triangle A R V$. If NO-ET homeostasis is in the pathological balance zone, antihypertensive treatment may yield high-level AUC and low-level $\triangle A R V$. If NO-ET homeostasis is in the pathological unbalanced zone, antihypertensive treatment may yield high-level AUC and high-level $\triangle A R V$. Therefore, it is recommended to administer antihypertensive treatment in the weak balance state.

\section{Simulation verification}

The results of experimental verification of the antihypertensive method are shown in Fig. 7. According to the treatment indication (Fig. 7a), macitentan should be given on the 4th day after sunitinib is administered. The high- and low-dose optimized treatment 


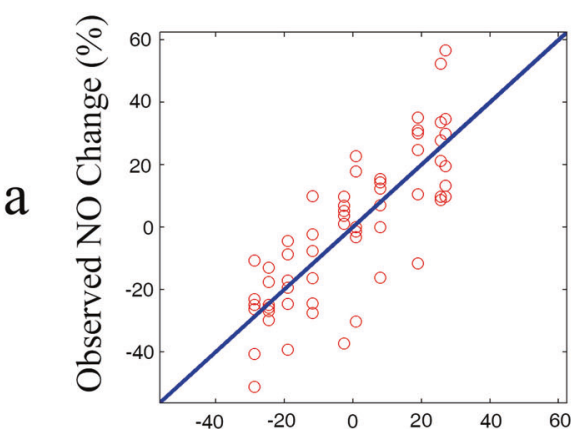

Predicted NO change $(\%)$
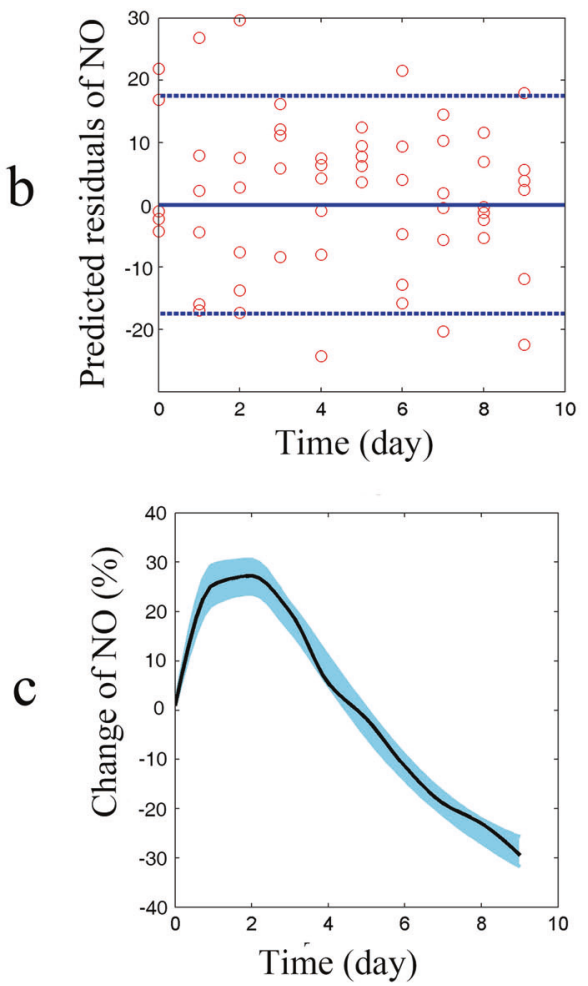

d

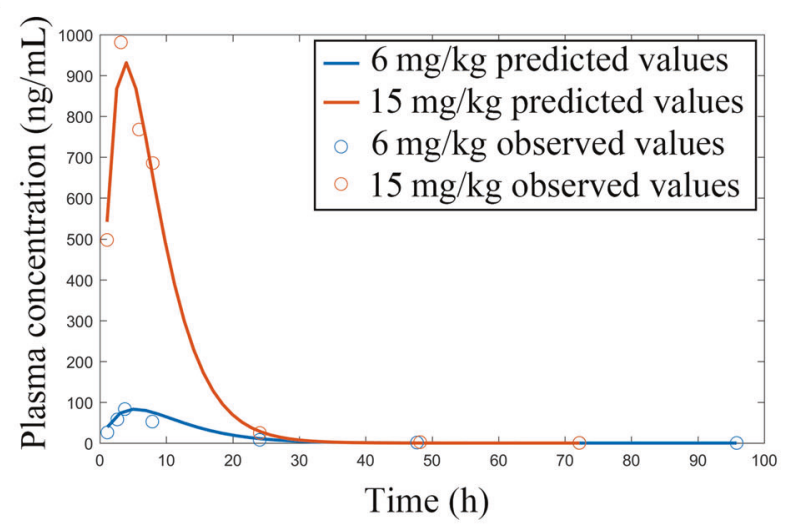

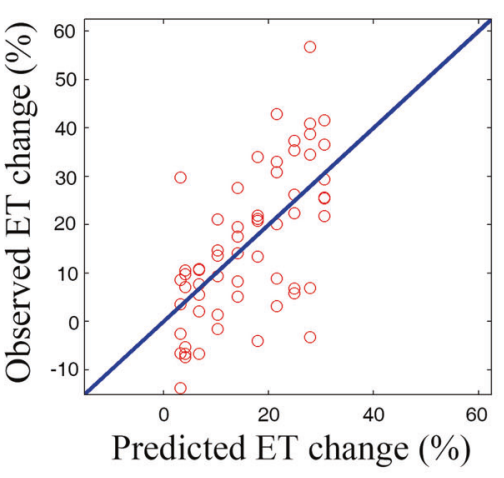
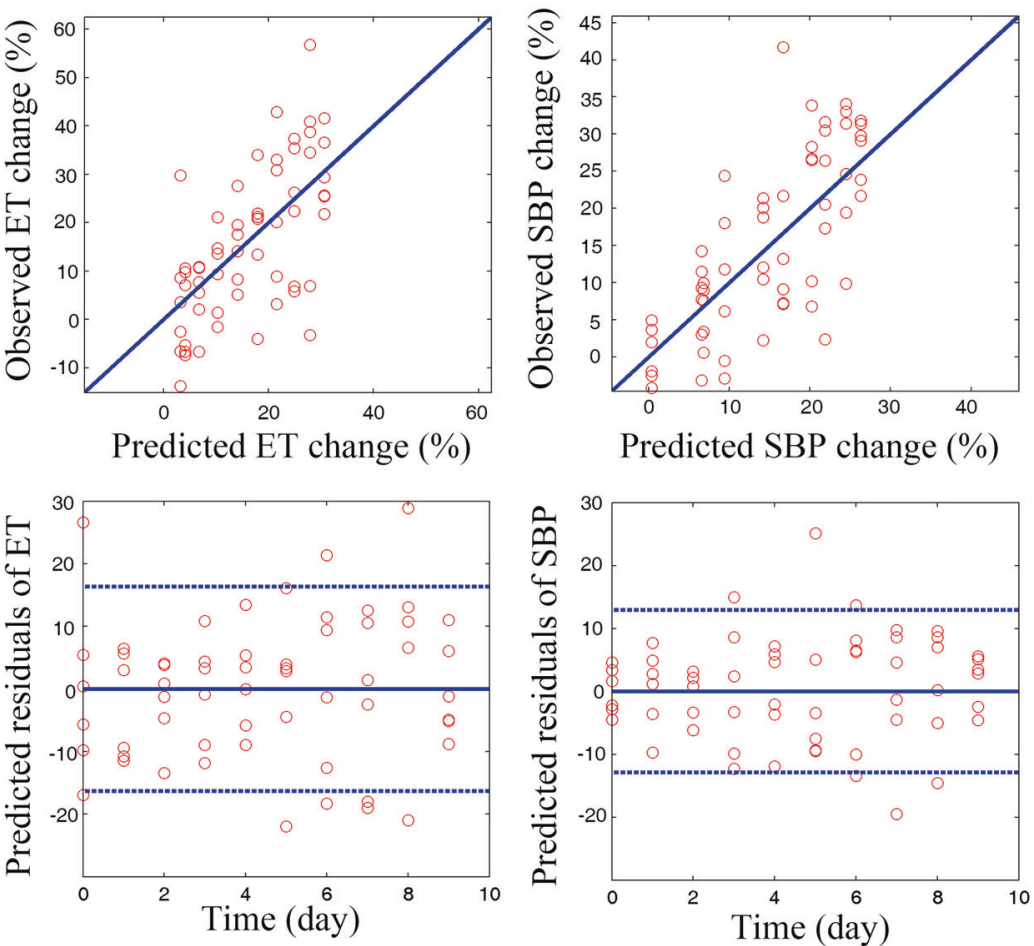

Predicted SBP change $(\%)$
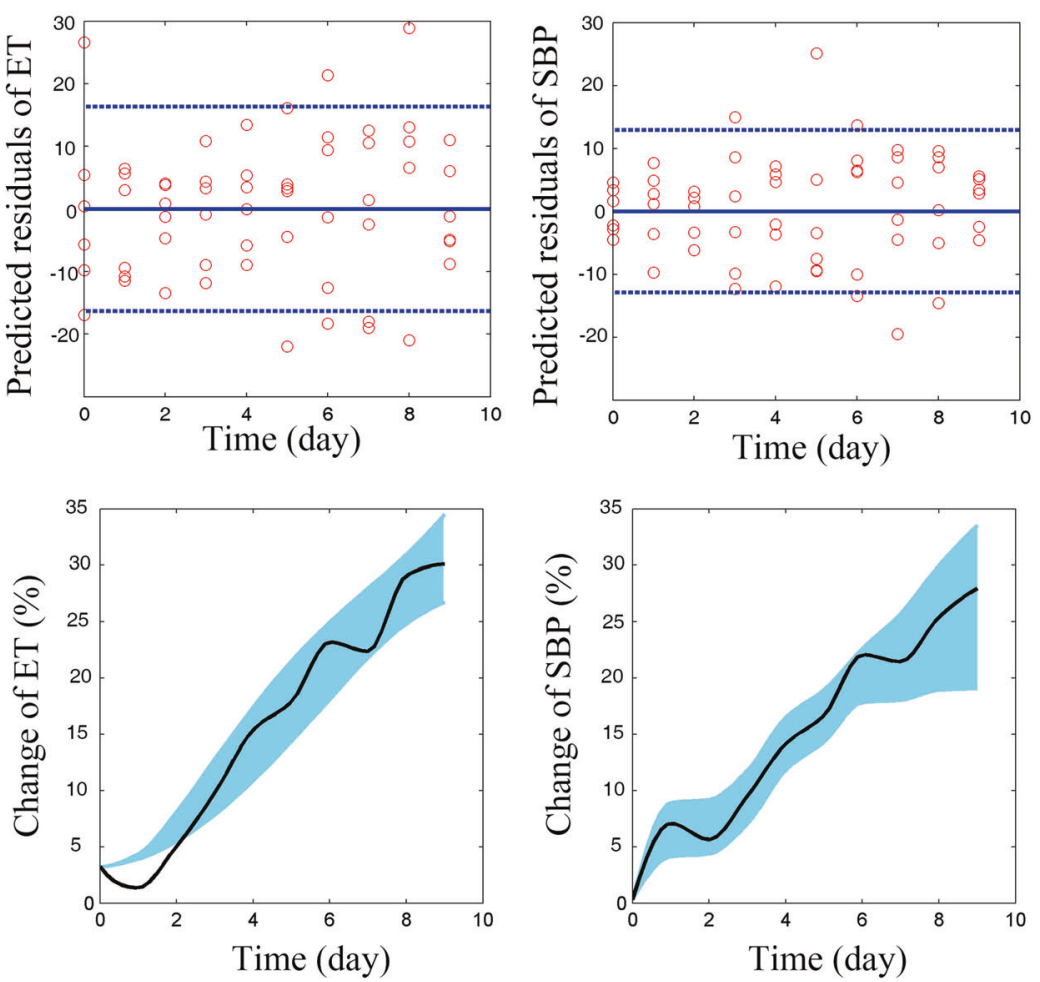

e

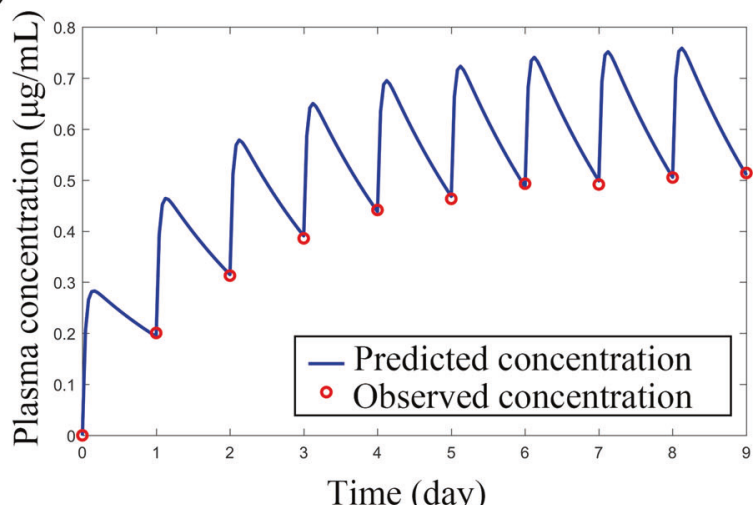

Fig. 3 Goodness-of-fit plots of the PK-PD model and the time course of sunitinib plasma concentration. a Scatter plots of observed values versus predicted values for NO, ET-1, and SBP. The line in each figure is a reference of perfect correlation (predicted value exactly equal to the observed value). b Scatter plots of predicted residuals vs. time for NO, ET-1, and SBP. c Visual predicted check (VPC) for NO, ET-1, and SBP. The shaded area represents the $95 \%$ confidence interval of the simulated median value. The line represents the median value of the observed value. $\mathbf{d}$ Single dose plasma concentration-time profiles of sunitinib e Multidose plasma concentration-time profiles of sunitinib. 

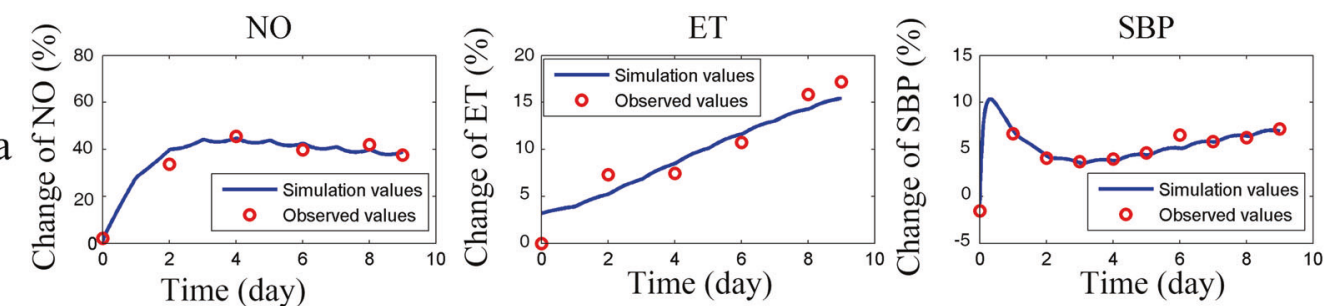

$\mathrm{b}$
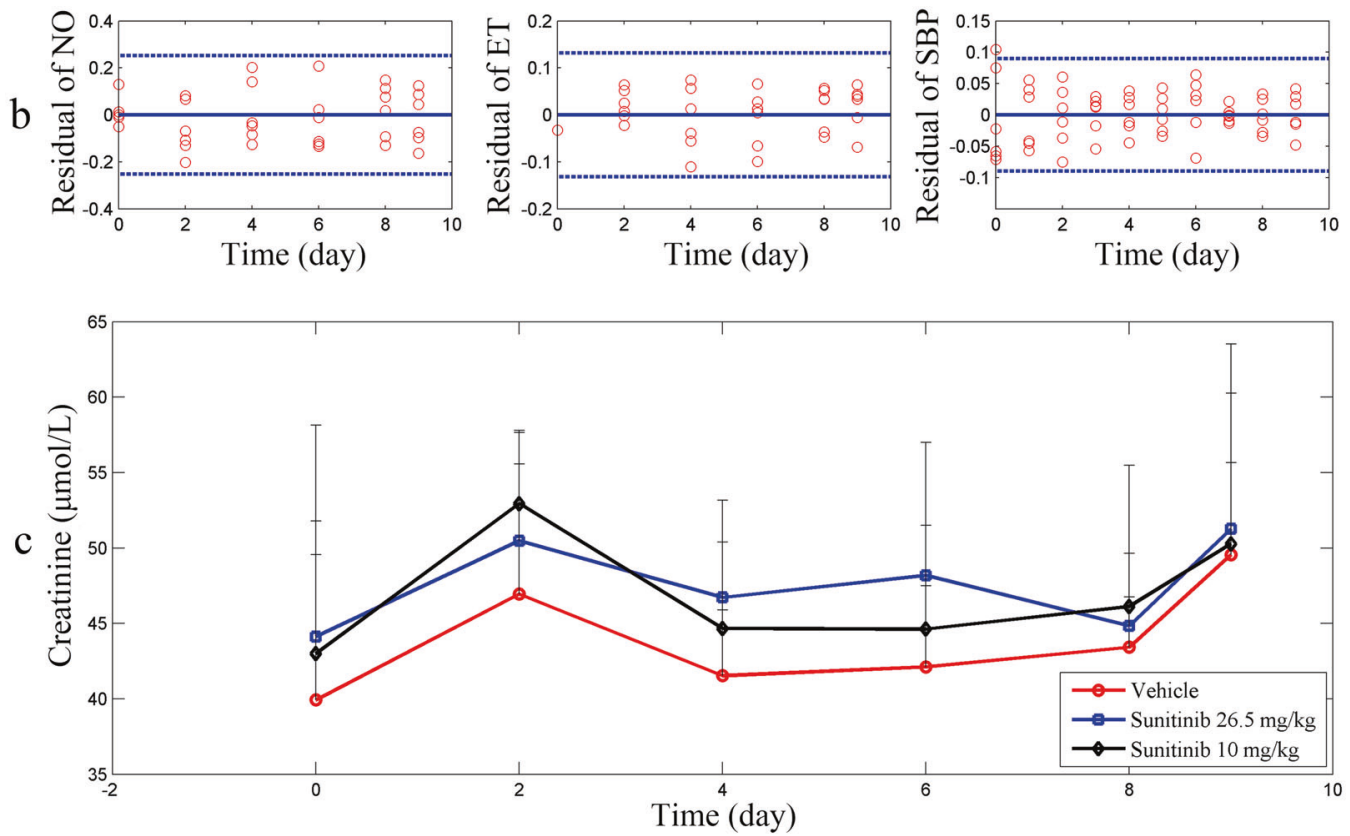

Fig. 4 External validation of the PK-PD model. a Simulated and observed time courses of NO, ET, and SBP. The circles represent the observed data for the low-dose group (sunitinib $10 \mathrm{mg} / \mathrm{kg}$ ). The line represents the simulation data for the PK-PD model. $\mathbf{b}$ Scatter plots of PK-PD model predicted residuals vs. time for NO, ET-1, and SBP of the low-dose group. c The creatinine level of the high-dose group (sunitinib $26.5 \mathrm{mg} / \mathrm{kg}$ ), low-dose group (sunitinib $10 \mathrm{mg} / \mathrm{kg}$ ) and control group (vehicle). The creatinine level was not significantly different among these three groups at any of the time points $(P>0.05$, based on one-way ANOVA).
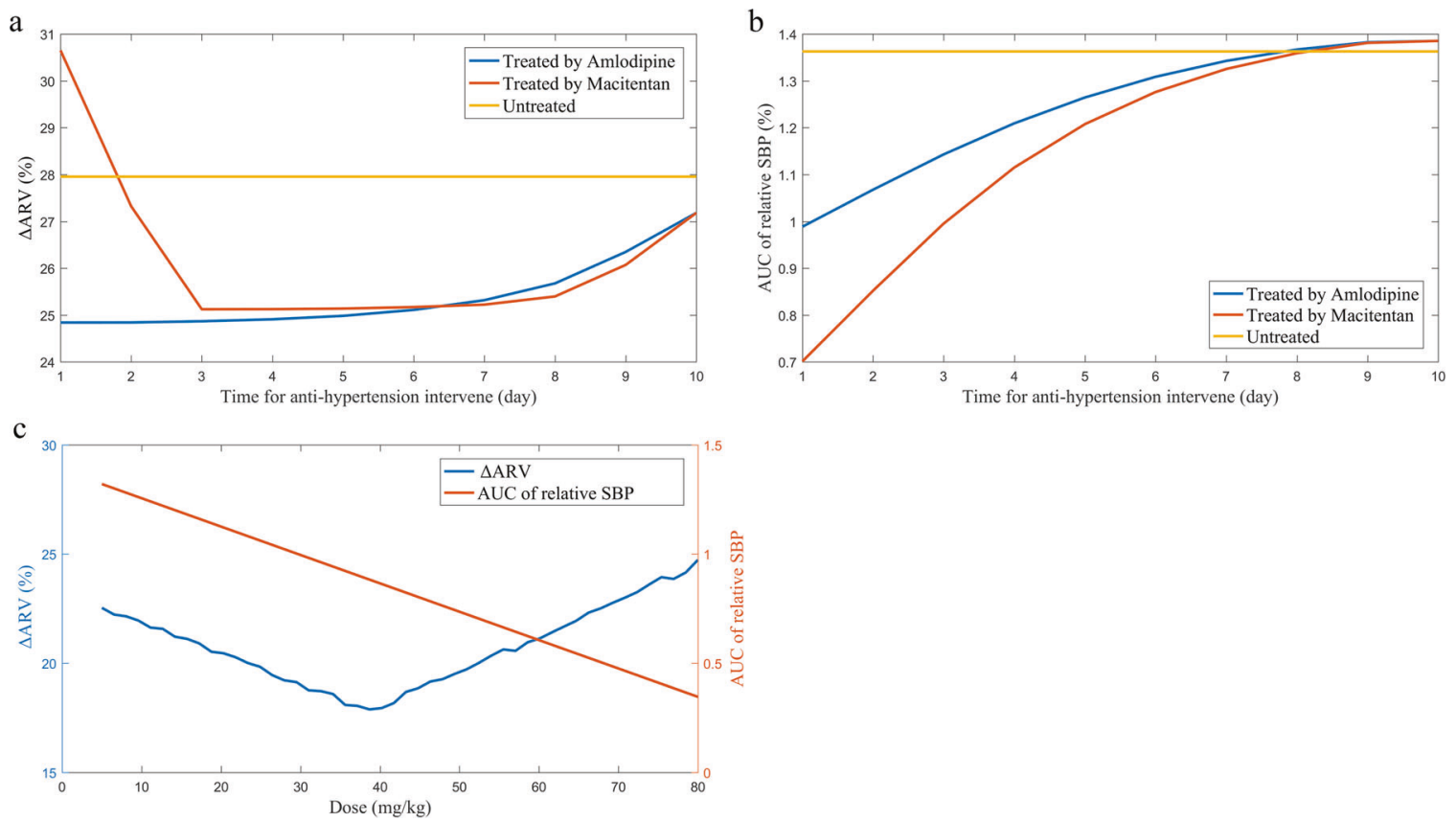

Fig. 5 Simulation results for scenarios I and II. ARV = average real variability. a The fluctuation of blood pressure (represented by relative $\mathrm{ARV}(\triangle \mathrm{ARV})$ ) yielded by amlodipine and macitentan treatment at different time points. $\mathbf{b}$ The AUC of blood pressure yielded by amlodipine and macitentan treatment at different time points. c The AUC and fluctuation of blood pressure (represented by relative ARV $(\Delta A R V)$ ) yielded by treatment with different doses of amlodipine and macitentan. 


\section{a}

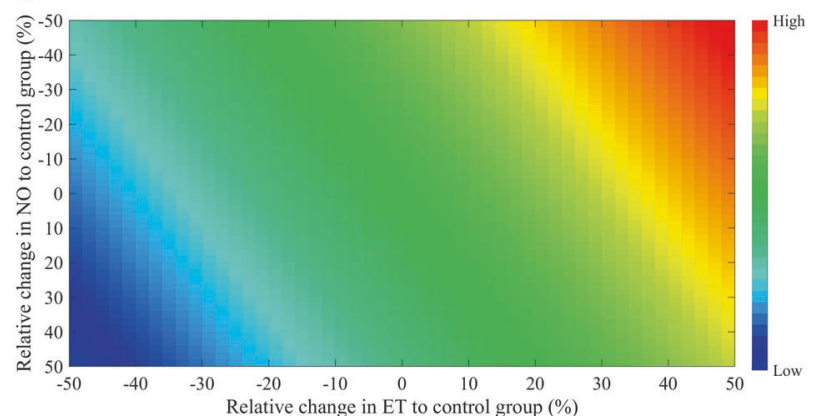

b

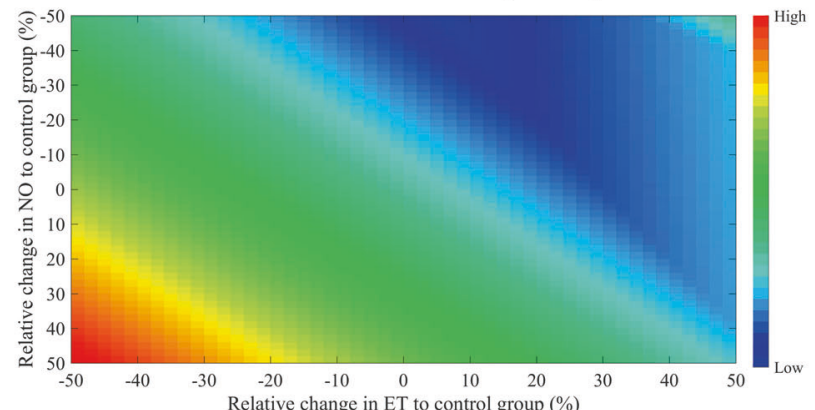

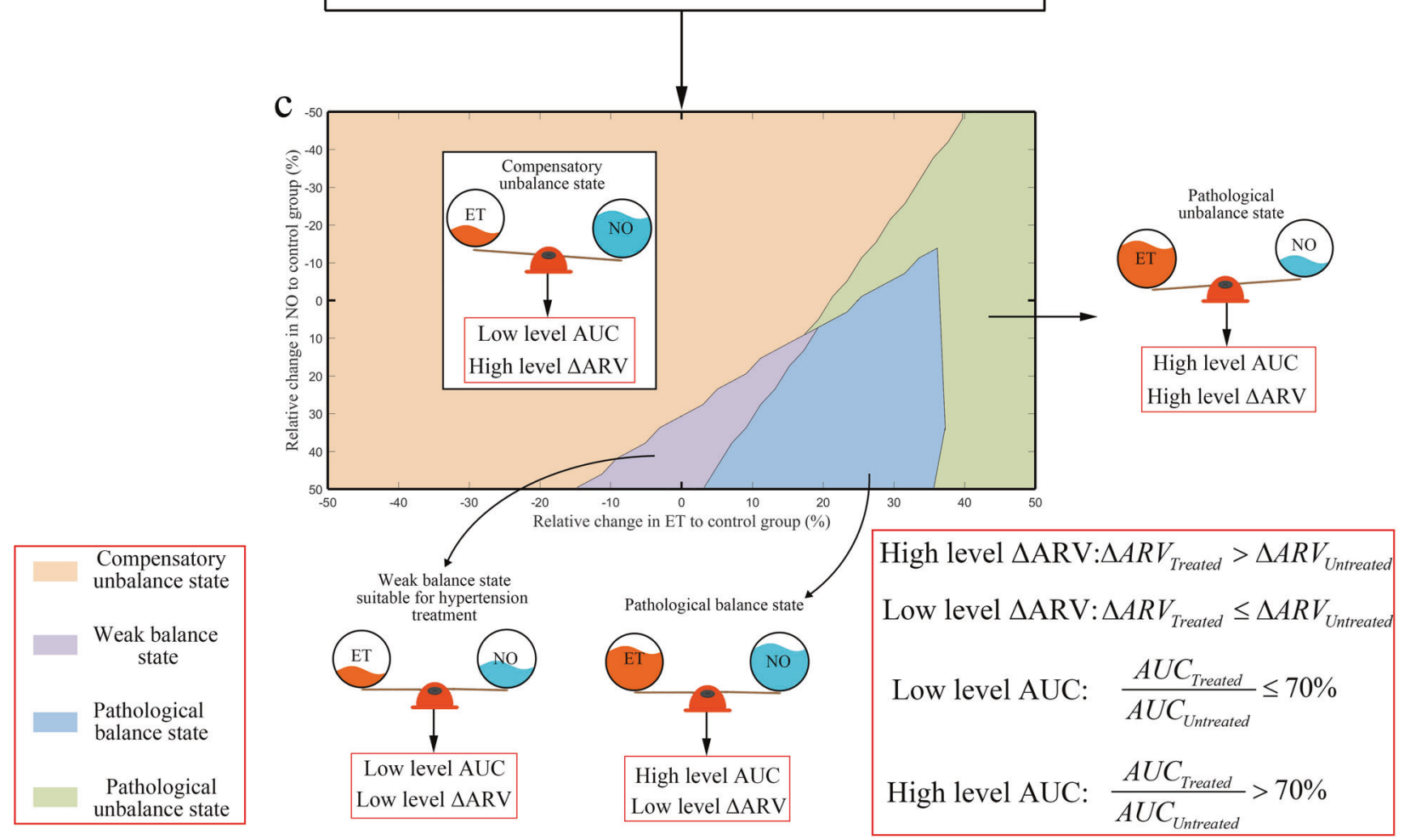
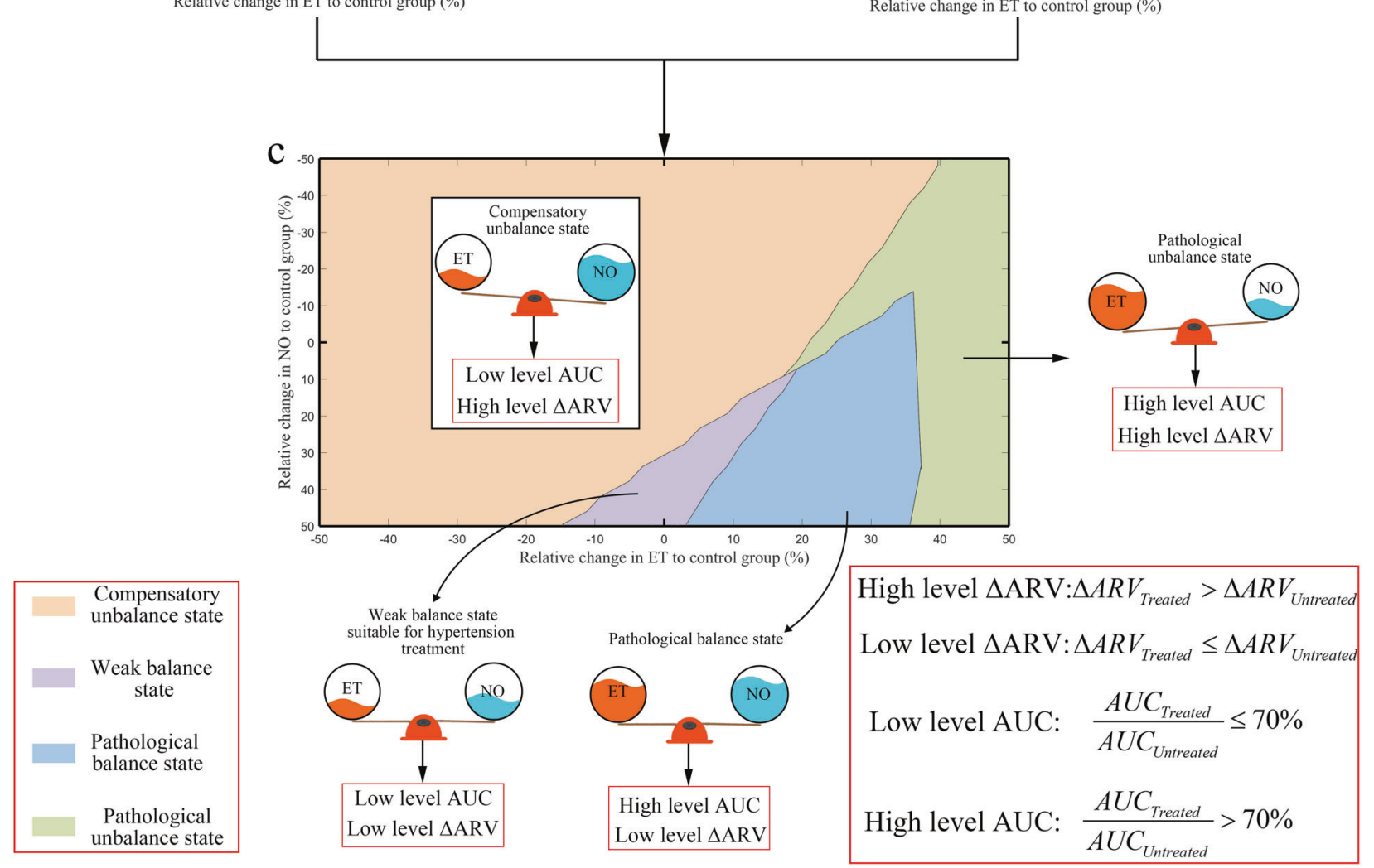

Fig. 6 Simulation results of scenario III. ARV = average real variability. a The AUC of SBP observed under different levels of ET and NO. b The fluctuation of SBP (represented by relative ARV $(\triangle A R V)$ ) observed under different levels of ET and NO. c The different response to antihypertensive treatment under different states of NO-ET homeostasis.

groups should be given $30 \mathrm{mg} / \mathrm{kg}$ macitentan and $10 \mathrm{mg} / \mathrm{kg}$ macitentan, respectively, at this time. The optimized treatment can lower AUC and blood pressure fluctuation dose-dependently (Fig. 7b). Compared with immediate antihypertensive treatment, optimized treatment can decrease $70 \%$ of blood pressure fluctuation.

\section{DISCUSSION}

In this study, a mechanism-based PK/PD model was developed to propose a method for the management of hypertension induced by sunitinib.

Previous studies have proposed that the mechanism of hypertension-induced sunitinib involves variations in NO-ET homeostasis. The NO system works in balance with the ET system, but sunitinib may decease NO, which makes NO unable to balance the vasoconstrictor ET [29-31]. Our study suggested that the balance between NO and ET may also affect the response of hypertension induced by sunitinib to antihypertensive agents. According to our experiment, from day 0 to day 3 after sunitinib administration, NO-ET homeostasis is in the compensatory unbalanced state. Previous research has shown that sunitinib may decrease NO, but our study showed that NO increased from day 0 to day 2 and then decreased [5]. The increase in ET-1 may stimulate NO release to balance the vasoconstrictive effect of ET-1 [23]. These results suggest that the system may still maintain NOET homeostasis in the compensatory unbalanced state. Subjects in this state may be highly sensitive to antihypertensive treatment. Our experiments and previous reports suggest that in the compensatory unbalanced state, antihypertensive treatment may lower blood pressure AUC $\left(A \cup C_{\text {Treated }} / A \cup C_{\text {Untreated }} \leq 70 \%\right)$ and may cause acute blood pressure fluctuation $\left(\triangle A R V_{\text {Treated }}>\right.$ $\left.\triangle A R V_{\text {Untreated }}\right)[3,6-8]$. Therefore, it is not recommended to administer any antihypertensive treatment in the compensatory unbalanced state because of the potential cardiovascular risk caused by blood pressure fluctuations. NO-ET homeostasis may enter the weak balance state on day 4 . In this state, the elevated sunitinib plasma concentration may enhance the inhibition of NO release, which may cause NO to decrease and limit the ability of NO-ET homeostasis to maintain blood pressure. Subjects in the 
a
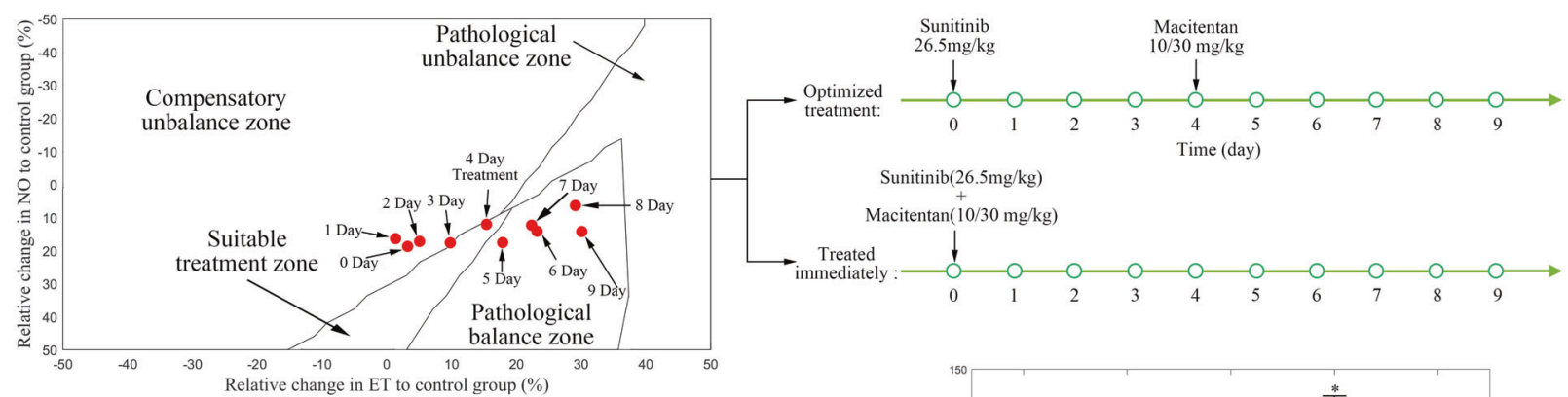

b
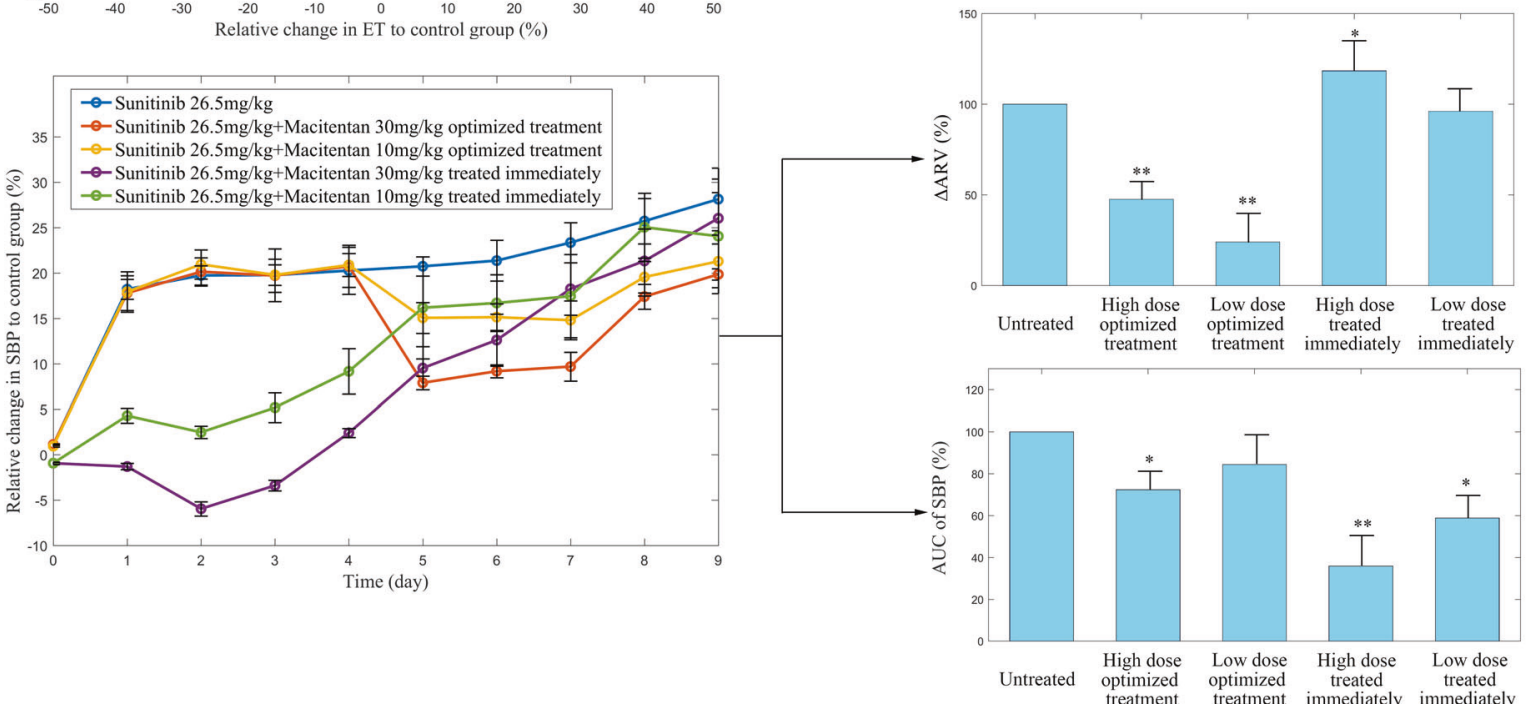

C

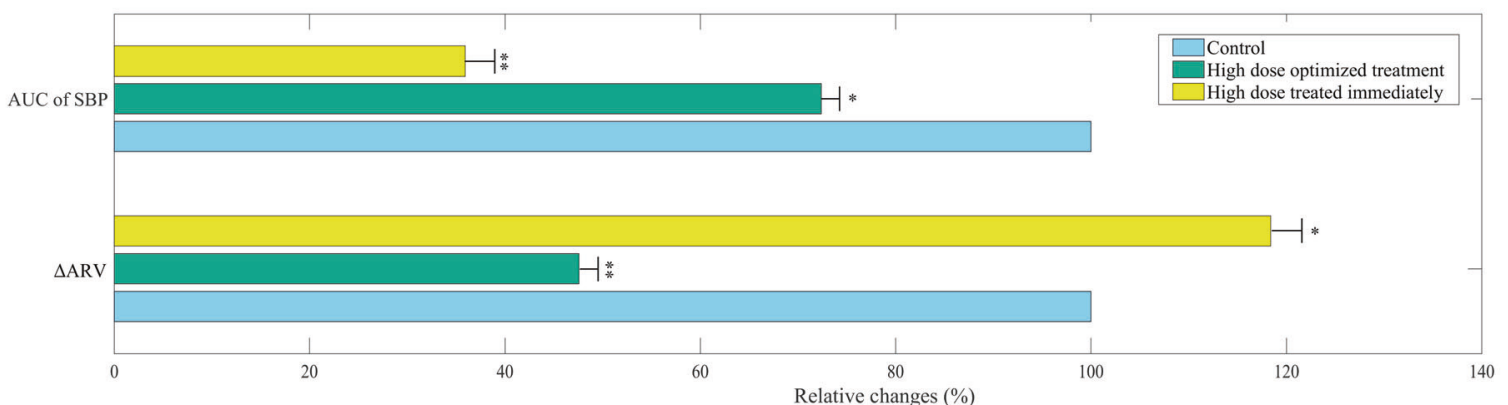

Fig. 7 Experimental results of validation of the simulation. ARV = average real variability. a the State of NO-ET homeostasis at different time points and the time point of antihypertensive treatment. $\mathbf{b}$ The results of SBP, SBP AUC and SBP fluctuation (represented by relative ARV $(\triangle A R V)$ ). c A comparison of the effects of optimized treatment and immediate treatment on AUC and the fluctuation of SBP (represented by relative $\operatorname{ARV}(\triangle \mathrm{ARV})) .{ }^{*} P<0.05,{ }^{* *} P<0.01$.

weak balance state may be moderately sensitive to antihypertensive treatment. Our simulations and experiments suggested that in this state, antihypertensive treatment can significantly lower AUC and blood pressure fluctuations $(P<0.01)$. Therefore, it is recommended to administer antihypertensive treatment in a weak balance state to reduce potential cardiovascular risk. From day 5 to day 9 after sunitinib administration, NO-ET homeostasis is in the pathological balance state. Compared with that in the weak balance state, sensitivity to antihypertensive agents decreases in this state. Our simulations showed that the effect of antihypertensive treatment in lowering blood pressure AUC may be limited but that it is still helpful in managing blood pressure fluctuations. In the pathological unbalanced state, SBP and ET-1 were markedly increased and NO decreased continuously. In this state, hypertension may be related to multiple other mechanisms because both the simulations and the previous research showed that the effect of the ET receptor antagonist macitentan is limited, which suggests that NO-ET homeostasis disorder may not be the sole causative factor of hypertension [3]. Apart from NO-ET homeostasis dysfunction, previous studies have proposed other hypotheses suggesting that decreased VEGF function leads to remodeling of capillary beds and endothelial dysfunction [31, 32]. As the mechanisms related to hypertension may be very complex, they remain to be further investigated. The potential translational value of the proposed model, after it is improved and validated by clinical data in the future, lies in providing a new method for optimizing the management of the hypertension induced by sunitinib.

\section{CONCLUSION}

In this study, a mechanism-based PK/PD model was developed to investigate a management method for hypertension induced by sunitinib. Our research suggested that it is recommended to use the state of the balance between ET and NO as an indicator for hypertension management. In this study, a novel mechanism- 
based model with potential translational value was developed for managing hypertension induced by sunitinib.

\section{ACKNOWLEDGEMENTS}

This study was supported by the National Natural Science Foundation of China (no. 81773806 and no. 81503145), "Double First-Class" University Project (no. CPU2018GY19) and the Fundamental Research Funds for the Central Universities (no. 2632018ZD10).

\section{AUTHOR CONTRIBUTIONS}

$\mathrm{HCL}, \mathrm{XTZ}, \mathrm{XQL}$, and $\mathrm{HH}$ designed the experiments. $\mathrm{HCL}, \mathrm{XTZ}$, and YSZ conducted the animal experiments. XTZ analyzed the samples. $\mathrm{HCL}$ developed the mathematic model and wrote the manuscript.

\section{ADDITIONAL INFORMATION}

Competing interests: The authors declare no competing interests.

\section{REFERENCES}

1. Hansson EK, Ma G, Amantea MA, French J, Milligan PA, Friberg LE, et al. PKPD modeling of predictors for adverse effects and overall survival in sunitinib-treated patients with GIST. CPT Pharmacomet Syst Pharm. 2013;2:e85.

2. Kappers MH, van Esch JH, Sleijfer S, Danser AH, van den Meiracker AH. Cardiovascular and renal toxicity during angiogenesis inhibition: clinical and mechanistic aspects. J Hypertens. 2009;27:2297-309.

3. Lankhorst S, Kappers MH, van Esch JH, Smedts FM, Sleijfer S, Mathijssen RH, et al. Treatment of hypertension and renal injury induced by the angiogenesis inhibitor sunitinib: preclinical study. Hypertension. 2014;64:1282-9.

4. Bamias A, Manios E, Karadimou A, Michas F, Lainakis G, Constantinidis C, et al. The use of 24-h ambulatory blood pressure monitoring (ABPM) during the first cycle of sunitinib improves the diagnostic accuracy and management of hypertension in patients with advanced renal cancer. Eur J Cancer. 2011; 47:1660-8.

5. Lankhorst S, Kappers MHW, van Esch JHM, Danser AHJ, van den Meiracker AH. Hypertension during vascular endothelial growth factor inhibition: focus on nitric oxide, endothelin-1, and oxidative stress. Antioxid Redox Signal. 2014; 20:135-45.

6. Grisk O, Koenen A, Meissner T, Donner A, Braun D, Steinbach A, et al. Rho kinase inhibition mitigates sunitinib-induced rise in arterial pressure and renal vascular resistance but not increased renal sodium reabsorption. J Hypertens. 2014;32: 2199-210.

7. Kappers MH, Smedts FM, Horn T, van Esch JH, Sleijfer S, Leijten F, et al. The vascular endothelial growth factor receptor inhibitor sunitinib causes a preeclampsia-like syndrome with activation of the endothelin system. Hypertension. 2011;58:295-302.

8. Witte J, Mühlbauer M, Braun D, Steinbach A, Golchert J, Rettig R, et al. Renal soluble guanylate cyclase is downregulated in sunitinib-induced hypertension. J Am Heart Assoc. 2018;7:e009557.

9. Matsui Y, Ishikawa J, Eguchi K, Shibasaki S, Shimada K, Kario K. Maximum value of home blood pressure a novel indicator of target organ damage in hypertension. Hypertension. 2011;57:1087-93.

10. Parati G, Ochoa JE, Lombardi C, Bilo G. Assessment and management of bloodpressure variability. Nat Rev Cardiol. 2013;10:143.

11. Hansson EK, Amantea MA, Westwood P, Milligan PA, Houk BE, French J, et al. PKPD modeling of VEGF, sVEGFR-2, SVEGFR-3, and SKIT as predictors of tumor dynamics and overall survival following sunitinib treatment in GIST. CPT Pharmacomet Syst Pharm. 2013;2:e84.
12. Kappers MH, van Esch JH, Sluiter W, Sleijfer S, Danser AH, van den Meiracker AH. Hypertension induced by the tyrosine kinase inhibitor sunitinib is associated with increased circulating endothelin-1 levels. Hypertension. 2010;56:675-81.

13. Lankhorst S, Baelde HJ, Kappers MH, Smedts FM, Hansen A, Clahsen-van Groningen $M C$, et al. Greater sensitivity of blood pressure than renal toxicity to tyrosine kinase receptor inhibition with sunitinib. Hypertension. 2015;66:543-9.

14. Thijs AM, van Herpen CM, Verweij V, Pertijs J, van den Broek PH, van der Graaf WT, et al. Impaired endothelium-dependent vasodilation does not initiate the development of sunitinib-associated hypertension. J Hypertens. 2015;33:2075-82.

15. Whitesall SE, Hoff JB, Vollmer AP, D'Alecy LG. Comparison of simultaneous measurement of mouse systolic arterial blood pressure by radiotelemetry and tail-cuff methods. Am J Physiol-Heart Circulatory Physiol. 2004;286:H2408-H15.

16. Kubota Y, Umegaki K, Kagota S, Tanaka N, Nakamura K, Kunitomo M, et al. Evaluation of blood pressure measured by tail-cuff methods (without heating) in spontaneously hypertensive rats. Biol Pharm Bull. 2006;29::1756-8.

17. Musijowski J, Piorkowska E, Rudzki PJ. Determination of sunitinib in human plasma using liquid chromatography coupled with mass spectrometry. J Sep Sci. 2014;37:2652-8.

18. Oberoi RK, Mittapalli RK, Fisher J, Elmquist WF. Sunitinib LC-MS/MS assay in mouse plasma and brain tissue: application in CNS distribution studies. Chromatographia. 2013;76:1657-65.

19. Moshage $H$, Kok $B$, Huizenga JR, Jansen PLM. Nitrite and nitrate determinations in plasma - a critical-evaluation. Clin Chem. 1995;41:892-6.

20. Speed B, Bu HZ, Pool WF, Peng GW, Wu EY, Patyna $S$, et al. Pharmacokinetics, distribution, and metabolism of $\mathrm{C}-14$ sunitinib in rats, monkeys, and humans. Drug Metab Disposition. 2012;40:539-55.

21. Cui W, Zhang ZJ, Hu SQ, Mak SH, Xu DP, Choi CL, et al. Sunitinib produces neuroprotective effect via inhibiting nitric oxide overproduction. CNS Neurosci Ther. 2014;20:244-52.

22. Eechoute K, van der Veldt AAM, Oosting S, Kappers MHW, Wessels JAM, Gelderblom $\mathrm{H}$, et al. Polymorphisms in endothelial nitric oxide synthase (eNOS) and vascular endothelial growth factor (VEGF) predict sunitinib-induced hypertension. Clin Pharmacol Ther. 2012;92:503-10.

23. Plato CF, Pollock DM, Garvin JL. Endothelin inhibits thick ascending limb chloride flux via ET(B) receptor-mediated NO release. Am J Physiol-Ren Physiol. 2000;279: F326-F33.

24. Lee JH, Kim EJ, Kwon JW, Yoo M, Lee MG. Negligible pharmacokinetic interaction between oral DA-8159, a new erectogenic, and amlodipine in rats. Biopharm Drug Dispos. 2006;27:125-31.

25. Bruderer S, Hopfgartner G, Seiberling M, Wank J, Sidharta PN, Treiber A, et al. Absorption, distribution, metabolism, and excretion of macitentan, a dual endothelin receptor antagonist, in humans. Xenobiotica. 2012;42:901-10.

26. Mena L, Pintos S, Queipo NV, Aizpurua JA, Maestre G, Sulbaran T. A reliable index for the prognostic significance of blood pressure variability. J Hypertens. 2005; 23:505-11.

27. de Miguel AG, Jimenez-Garcia R, San Martin M, Gonzalez IF, Campo AV, Gonzalez $J M$, et al. Drug surveillance study of amlodipine in patients with hypertension not controlled with drug therapy: NORCON study. Curr Ther Res-Clin Exp. 2000; 61:863-70.

28. Sidharta PN, van Giersbergen PL, Halabi A, Dingemanse J. Macitentan: entry-intohumans study with a new endothelin receptor antagonist. Eur J Clin Pharmacol. 2011;67:977-84.

29. Dhaun N, Webb DJ. Receptor tyrosine kinase inhibition, hypertension, and proteinuria: is endothelin the smoking gun? Hypertension. 2010;56:575-7.

30. Dhaun N, Goddard J, Kohan DE, Pollock DM, Schiffrin EL, Webb DJ. Role of endothelin-1 in clinical hypertension: 20 years on. Hypertension. 2008;52:452-9.

31. Fishbein L, Cohen DL. Systemic therapies for malignant pheochromocytoma and paraganglioma can exacerbate hypertension. J Clin Hypertens. 2013;15:513-4.

32. Aparicio-Gallego G, Afonso-Afonso FJ, Leon-Mateos L, Firvida-Perez JL, VazquezEstevez S, Lazaro-Quintela $M$, et al. Molecular basis of hypertension side effects induced by sunitinib. Anticancer Drugs. 2011;22:1-8. 